\title{
Creación de una estrategia didáctica de investigación basada en el diálogo de saberes
}

- CREATION OF A DIDACTIC INVESTIGATION STRATEGY BASED ON KNOWLEGDE DIALOGUE

- CRIAÇÃO ESTRATÉGICA DIDATICA DE PESQUISA BASEADA NO DIALOGO DOS SABERES

Mónica Moreno Torres* / luna3910@hotmail.com

\section{Resumen}

El texto expone los resultados de una investigación que se pregunta por la posibilidad de construir una estrategia didáctica de investigación basada en el diálogo de saberes. Por ello, propone y expone la creación de una Espiral Comprensiva de la Experiencia de Investigación en el Aula - ECEDIA-, en cuyo interior se encuentran diez componentes que articulan los saberes del docente (disciplinar y didáctico) a los aprendizajes de investigación de sus estudiantes.

\section{Summary}

The text presents the results of research that questions the possibility of building a teaching strategy based on research knowledge dialogue. Therefore proposes and discusses the creation of a Spiral Comprehensive Research Experience in the Classroom- ECEDIA-, inside which there are ten components that articulate teacher knowledge (disciplinary and didactic) to learning research of their students.

\section{Resumo}

O presente texto expõe os resultados de uma pesquisa em que se questionam as possibilidades de construir uma estratégia didática em pesquisa baseada no dialogo de saberes. Por isso, propõe e expõe a criação de uma Espiral Compreensiva da Experiência de Pesquisa em sala de aula - ECEDIA-, que cujo interior encontram-se dez componentes que articulam os saberes do professor (disciplinar e didático) às aprendizagens de pesquisa dos seus estudantes.

\section{Palabras clave}

Diálogo de saberes, conversación hermenéutica, hecho sorprendente, hipótesis abductiva, evaluación dialógica, investigación dialógica.

\section{Keywords}

Knowledge dialogue, hermeneutic conversation, surprising fact, abductive hypotheses, dialogical assessment, dialogical research.

\section{Palavras chaves}

Diálogo dos saberes, conversação hermenêutica, feito surpreendente, hipótese abdutiva, avaliação dialógica, pesquisa dialógica.

* Profesora de la Facultad de Educación de la Universidad de Antioquia. Magister en Literatura Colombiana de la Facultad de Comunicaciones de la Universidad de Antioquia. Doctoranda de la línea, Didáctica de la Educación Superior, de la Facultad de Educación de la Universidad de Antioquia. 


\section{Introducción}

Los procesos de investigación en el aula, bien sea en la educación básica, media o superior, presentan una serie de dificultades, entre las que es dable destacar la desarticulación entre el saber disciplinar, el saber didáctico y los aprendizajes de investigación de los estudiantes. Estos últimos, pueden estar motivados por responder a una pregunta o un problema de investigación, pero cuando su tratamiento se focaliza en la verificación de fuentes externas, o en el reporte de informes orales y escritos, como un resultado del proceso, se desconocen las posibilidades que ofrece el diálogo de saberes entre el docente y sus estudiantes dentro de la reconstrucción de estos conocimientos .

A esto se suma la elección del tema-problema objeto de investigación. En algunos casos, el docente es quien toma la iniciativa, en otras ocasiones, los estudiantes pueden encontrar un tema de su interés, pero cuando las estrategias didácticas del docente carecen de algunos elementos clave que garanticen la permanencia del Deseo de saber que los estudiantes expresan de manera natural al comienzo de su investigación, esta última no se culmina. Dar a la investigación en el aula el carácter de un proceso significativo para los estudiantes de la educación básica y media, exige al docente, tal como lo expresa Balbi (2008), inscribirse en un enfoque constructivista de la investigación. En esta perspectiva, la actividad del docente (y del estudiante), puede convertirse en un ejercicio de investigación basado en la selección de unos contenidos y unas estrategias inspiradas en la formulación de preguntas, problemas y proyectos.

En este mismo sentido, García y García (1993), consideran que si la investigación cualitativa en el aula tiene como punto de partida la red conceptual de la disciplina enseñada por el docente, éstas serían algunas de sus características: el problema de investigación formulado por los estudiantes podría surgir a modo de hipótesis; el proceso de investigación estaría representado por medio de "una organización en espiral" basada en dos dimensiones, una dimensión horizontal en la que se encontraría la red conceptual, y otra dimensión vertical, cuyo movimiento expresaría la profundización creciente que podrían adquieren de cada concepto los estudiantes.

Por su parte, González (2005), señala que el desarrollo de competencias investigativas de los estudiantes en la educación superior, es uno de los propósitos del docente en la universidad, pues su cognoscibilidad científica debe convertirse en una de las variables de su método didáctico. Dichas competencias incluyen las siguientes habilidades: inducción, deducción y abducción, con sus respectivos sistemas de competencias. Estas habilidades promueven la alta inteligencia y se convierten en el camino metódico que debe ser simulado didácticamente en el aula.

Esta idea de construir una estrategia didáctica interesada en promover algunas habilidades de investigación en los estudiantes, es consecuente con lo expresado por Ruíz y Torres (2005), quienes cuestionan la presencia de asignaturas como "métodos de investigación" y espacios de conceptualización afines a éstos, pues diversos estudios han demostrado que los profesores tienden a transmitir información relacionada con los métodos de investigación, dejando de lado el desarrollo de la competencia investigativa de los estudiantes.

En suma, los procesos de investigación en el aula (básica, media y superior), comienzan a mostrar que es posible pensar en modelos o estrategias didácticas de investigación, que articulen las preocupaciones teóricas del profesorado (saber disciplinar y saber didáctico) a los aprendizajes de investigación de sus estudiantes. Con base en esta intencionalidad, se construyeron los siguientes principios de la estrategia didáctica, representada en la Espiral Comprensiva de la Experiencia de Investigación en el Aula - ECEDIA-: la investigación es una experiencia dialógica, debido a su carácter interhumano y heurístico; la interacción pedagógica se potencia por medio de la conversación hermenéutica, debido a su interés en articular las expectativas de los estudiantes con los propósitos educativos y didácticos del docente y la sociedad; y el conocimiento de la ciencia y la cultura, al estar basado en el diálogo de saberes, le permite al docente asumirse como un práctico reflexivo de los saberes profesionales que comparte con sus estudiantes, sus colegas y la sociedad.

\section{Metodología}

Estos principios se inspiran en la investigación cualitativa con enfoque hermenéutico. En este enfoque, investigadoras sociales, como Gurdián (2007), y cualitativas, como González (2010), proponen la inclusión de la hermenéutica en la investigación en educación. La primera, hace un Ilamado al docente de educación 
básica y media para que supere el enfoque positivista de la investigación en el aula1; y la segunda, diseña un modelo de investigación cualitativa con enfoque hermenéutico para el docente universitario.

De esta manera, Gurdián (2007) considera que en la década de 1970 se desarrollan de manera significativa una serie de corrientes teórico-metodológicas en el ámbito de las ciencias sociales y humanas, entre las que se destaca una concepción hermenéutica de la ciencia Ilamada "paradigma hermenéutico", cuyos enfoques más representativos son el etnográfico, el sociológico, el interpretativo y el interaccionismo simbólico, entre otros.

Para estos enfoques, el método hermenéutico nos permite identificar y definir los siguientes componentes de la investigación: el contexto de la indagación, el tema u objeto de estudio, las técnicas para la recolección y el análisis de la información. Así, mientras el método conserva su carácter de procedimiento, la metodología ha de "transformarse en una disciplina que estudia, analiza, promueve y depura el método mismo" (p. 134).

Algunos conceptos clave de este enfoque son: concibe el conocimiento como una construcción colectiva e histórica; el intercambio de saberes es dialéctico; se apoya en el arte de la pregunta para avanzar en dicho intercambio; los prejuicios, las creencias, los valores y los mitos, son unidades de sentido encaminadas a la producción de conocimiento; la interpretación de sentido se realiza desde "la otredad", pues se interesa por mantener las relaciones entre lo objetivo y lo subjetivo; el investigador se desplaza de las partes al todo y del todo a las partes; entre otros.

En consonancia con lo anterior, González (2010) considera que la experiencia hermenéutica tiene como fundamento un proceso, una estructura y un procedimiento. El proceso lo determinan los prejuicios, la reflexión, el análisis, la comprensión, la interpretación y la síntesis. Estos componentes dinamizan el procedimiento y la estructura. La estructura está determinada por los componentes mencionados, y el procedimiento incluye un problema dialéctico, una hipótesis abductiva, la historia de conceptos, el estado en cuestión y la conversación con las autoridades en el tema investigado para llegar al

1 Uno de los propósitos de Gurdián con su obra El paradigma cualitativo en la investigación socio-educativa, consiste en familiarizar al docente con el enfoque cualitativo y mostrarle que dicha teoría rompe con los modelos tradicionales (positivistas) de la investigación en el aula (2007: $34)$. acuerdo de la cosa. Estos fundamentos producen una unidad de sentido que le otorga legitimidad a lo creado.

El valor de la hipótesis abductiva en este procedimiento tiene dos funciones: "mientras el sujeto se pregunta, responde, y pregunta en tanto duda", y duda al intuir la presencia de la cosa a crear, la hipótesis promueve el surgimiento de nuevas preguntas como una posibilidad para avanzar en su comprensión; participa de un juego intersemiótico creado por un hecho sorprendente. Este hecho es el primer detonador de la duda y de las preguntas, se convierte en la anticipación de sentidos de la cosa a crear, y puede entenderse como un signo que ingresa al círculo de la comprensión, encargado de darle sentido a las partes y el todo de la investigación.

La hipótesis abductiva de esta investigación es la siguiente: ¿Es posible traducir la teoría de la abducción y la hermenéutica [literaria], en una estrategia didáctica basada en el diálogo de saberes para la formación de profesores investigadores en la educación básica, media y superior?2.

Cuando decimos hermenéutica, reconocemos sus aportes a la investigación cualitativa, tal como lo mostramos en la perspectiva de Gurdián (2007) y González (2010); al ponerla en relación con la literatura, ubicamos sus aportes a la hermenéutica literaria y a la Didáctica de la literatura, esta última y la Didáctica universitaria, son los objetos de estudio de esta investigación. En el caso de la Didáctica de la literatura, se analizan los marcos teóricos y los enfoques que la conciben como un estímulo a los procesos de investigación en el aula. Esta intención, se proyecta en la interacción que la proponente de esta investigación adelanta con diversos grupos de maestros en formación, del área de lengua y literatura de la Facultad de Educación de la Universidad de Antioquia.

Estos grupos se convirtieron en los primeros interlocutores de la ECEDIA, en tanto su diseño y desarrollo fue valorado por los maestros en formación, cada vez que interactuaban con los siguientes instrumentos metodológicos de la investigación: el diseño y la puesta en escena de talleres, apoyados en la lectura predictiva de relatos enigmáti-

\footnotetext{
2 En la visión peirceana, formular una abducción en forma interrogativa nos permite mostrar que si dudamos de algo debemos ponerlo en cuestión, mientras probemos una conclusión que nos acerque a la verdad del hecho (Peirce, 1901). Para la hermenéutica y la teoría de la abducción, las conclusiones y la verdad son transitorias, pues el conocimiento siempre se encuentra en evolución.
} 
cos y policiacos; la construcción de proyectos de aula para la educación básica y media, pues los maestros en formación también adelantaron procesos de planeación y de investigación inspirados en la formulación de un hecho sorprendente $y$, posteriormente, en una hipótesis abductiva ${ }^{3}$.

En el caso de la Didáctica universitaria, se estudian los problemas teórico-prácticos a los que se enfrenta este campo de conocimientos, y se adelantan las siguientes experiencias de aula: en Octubre y Noviembre de 2010, la Universidad de Medellín — privada_ y la Universidad de Antioquia — pública - realizaron un Diplomado en fundamentación pedagógica y didáctica. La primera institución convocó a treinta profesores de cátedra de las siguientes áreas: sociales, ingeniería, ciencias económicas y de la comunicación, entre otras. La segunda, promovió la participación de treinta profesores de cátedra adscritos al Departamento de Trabajo Social de su Facultad de Ciencias Sociales y Humanas.

Un análisis comparativo de estas experiencias, señala lo siguiente: el primer taller, realizado en la Universidad de Medellín, se organizó alrededor de una guía semioepistemológica, mostrando un mayor énfasis en el marco teórico de esta investigación. A diferencia del segundo, que si bien tiene en cuenta la misma teoría, intenta superar la mediación didáctica de la primera al darle un mayor énfasis al concepto "taller" 4 . En ambas experiencias se conserva el "horizonte literario", sin embargo, en la segunda se habla de la "fusión horizóntica de saberes: disciplinar y didáctico".

Este segundo taller presenta un salto cualitativo respecto del primero, en tanto que hay una mayor conciencia de la proponente de esta investigación por traducir la teoría de la abducción y la gadameriana, en una estrategia di-

3 Los proyectos de investigación en el aula desarrollados por los futuros maestros, comprenden de 2007 a 2009. Dichos proyectos, se adelantaron en los cursos Didáctica de la lengua y la literatura II, de la Licenciatura en Humanidades, Lengua Castellana, de la Facultad de Educación de la Universidad de Antioquia.

4 En el taller no hay programas preestablecidos, pues se trata de un juego conjetural mediatizado por la formulación de un hecho sorprendente, producto de la capacidad de intuición e imaginación del docente y sus estudiantes. Después del hecho sorprendente, viene la hipótesis, formulada a partir de un tema-problema, relacionado con el saber disciplinar y el saber didáctico del profesor. dáctica que promueva los siguientes procesos: el diálogo de saberes entre los profesores y de estos últimos con la proponente; y la articulación entre las dimensiones pedagógica, didáctica y disciplinar, con la postura éticopolítica e investigativa de los profesores de esta institución.

Estos cambios posibilitaron la construcción de una estrategia didáctica, representada en la ECEDIA, que además de tener sentido para los maestros en formación del área de lengua y literatura, se convierte en un aporte a los procesos de investigación en la educación superior. Los componentes de la ECEDIA están representados por una serie de nodos conceptuales que, en la perspectiva de Maturana (1997, p. 53), podrían entenderse como una serie de "coordinaciones consensuales y distinciones", propias de un sistema biológico y social.

Así las cosas, el ingreso del docente y sus estudiantes a la ciencia, la cultura en general y la cultura académica en particular, son "caminos de la sabiduría y de la comprensión que deben ser cultivados si es que han de tener alguna presencia en nuestra vida diaria" (Maturana, 1997, p. 90). Una manera de avanzar en este camino podría estar representada en el desarrollo de la - ECEDIA-, que exponemos a continuación.

\section{Resultados}

Uno de los hallazgos de esta investigación está representado en el siguiente mapa mental de la ECEDIA (Figura 1):

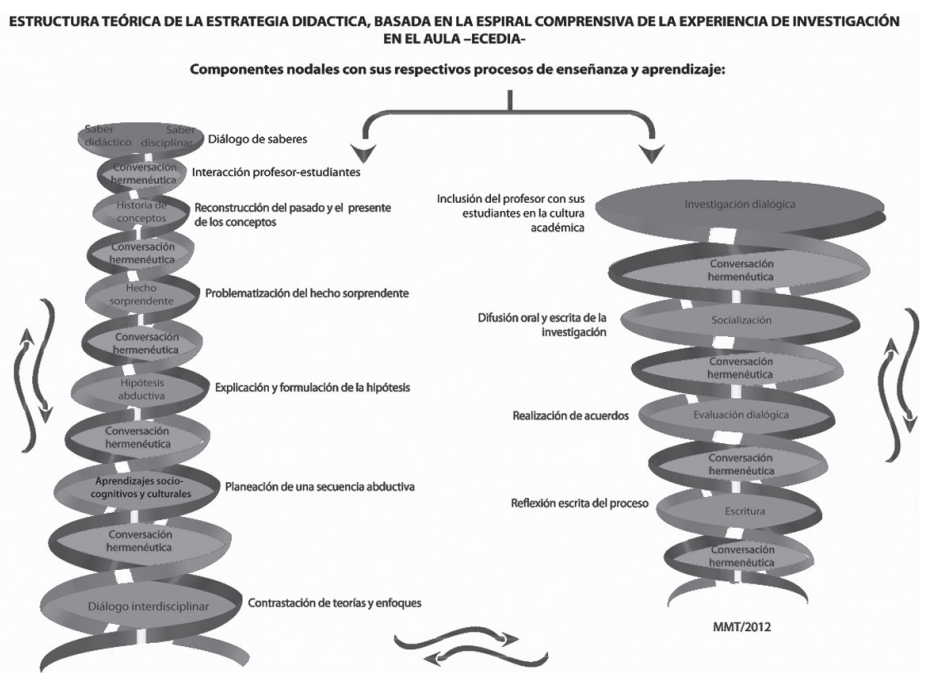

Figura 1. Mapa mental de la ECEDIA 


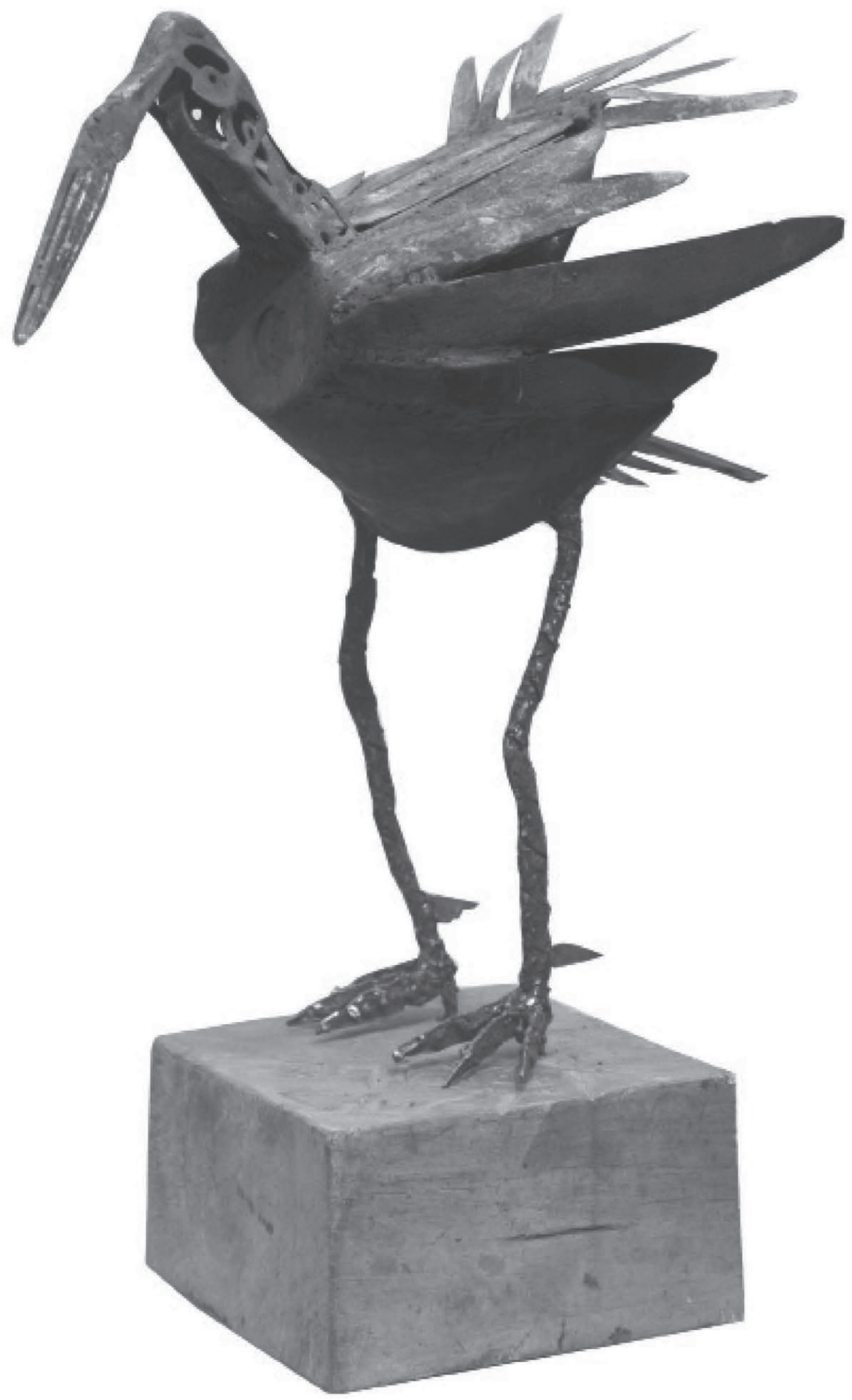

Artista: Felipe Ruiz

Titulo: Gallina

Dimensiones: $50 \times 35 \times 30 \mathrm{~cm}$

Tecnica: Escultura en metal

Año: 2012 
Con base en lo anterior, miremos de manera sintética cada uno de los componentes de la ECEDIA.

\section{A. El diálogo de saberes}

El docente es un traductor de saberes al hacer enseñables los conocimientos que provienen de la ciencia y la cultura; los problemas de la ciencia y la cultura son un producto social y político que debe ser analizado por el docente con sus estudiantes; si la ciencia es un proceso en constante evolución, sus hallazgos deben ser discutidos con la idea de actualizar su pasado y proyectarlo hacia un futuro digno para los ciudadanos del planeta; el trabajo dialógico entre el docente y sus estudiantes, y de estos últimos con otras comunidades académicas, educativas, de aprendizaje y virtuales, debe contribuir con la consecución de sus aspiraciones individuales y colectivas.

\section{B. La conversación hermenéutica}

Este componente, es el eje transversal de la estrategia didáctica y su ECEDIA, pues el diálogo de saberes sería impensable sin la palabra hablada y escrita al interior del salón de clases. Hablada, debido a las interacciones permanentes entre el profesor con sus estudiantes y de estos últimos con sus pares (el grupo-clase y la cultura académica), en busca de un acuerdo. Escrita, pues ésta se convierte en un espacio para la autorreflexión y el diálogo del sujeto con la cultura (textos científicos y literarios) y sus compañeros de equipo de investigación 5 .

\section{Historia de conceptos}

De acuerdo con Gadamer (2005), el investigador que reconoce el valor de la tradición está motivado por el presente de la cosa y por unos intereses especiales; es precisamente ese interés el mecanismo que le permite motivarse a construir un tema y un objeto de investigación. El interés histórico por la cosa investigada no está en el objeto, pues no se trata de comprender el objeto per se, sino de reconocer "el aspecto bajo el cual se nos muestra" en la experiencia hermenéutica. En este caso,

5 Este nodo conceptual de la ECEDIA, se inspira en algunas reflexiones de Gadamer (2004), como por ejemplo, cuando se pregunta si “¿está desapareciendo el arte de la conversación?" (p. 203). En otra de sus obras, Gadamer (2005), aborda el concepto de la precomprensión y lo pone en relación con las tensiones que se presentan entre las ideas propias y las ajenas, distinción fundamental para la formación del espíritu crítico de los estudiantes. se trata de una vivencia que se dirige hacia un objeto, una estrategia didáctica. Por eso, cuando decidimos estudiar la historia del concepto "experiencia" lo consideramos un elemento clave de la hermenéutica, la literatura, la didáctica y la investigación en el aula, debido a su relación con los procesos de interacción humana ${ }^{6}$.

\section{Hecho sorprendente}

El hecho sorprendente se convierte en el primer detonador abductivo de la investigación en el aula. Una manera de posibilitar su surgimiento consiste en proponer a los estudiantes una lectura abductiva de un artículo de investigación (en la universidad) y un relato enigmático (en la educación básica y media). En el relato enigmático, el lector participa del juego conjetural cuando se interesa por descifrar una fechoría (crimen, pérdida de un objeto clave para esclarecer el problema narrativo, la deshonra de un personaje, entre otros), mientras que en el artículo de investigación, las hipótesis, las preguntas y las conclusiones del texto se pueden convertir en marcas textuales abductivas. En palabras de Nubiola (2001, p. 12):

[...] la comprensión de la estructura lógica de toda abducción se encierra en el carácter sorprendente del hecho referido en la primera premisa, y en el trabajo de la imaginación en la segunda, cuando descubre que si determinada hipótesis fuera verdadera, convertiría el hecho sorprendente en un acontecimiento normal, razonable, y por tanto no sorprendente.

Lo anterior, recomienda que si bien un hecho puede aparecer como algo sorprendente en un sujeto (un estudiante), es posible que su epifanía (iluminación sorpresiva), al ser imaginada y ponerse en relación con una serie de premisas o argumentos, demuestre que no existe tal abducción. Esta advertencia no se puede convertir en un obstáculo para la investigación en el aula, pues la ciencia, la cultura y la educación, son procesos sociales en constante evolución.

\section{E. Hipótesis abductiva}

De acuerdo con Peirce (1901), la formulación de una hipótesis comprende tres momentos: la invención, selección y consideración de la hipótesis, proceso que equivale a la

6 Para una ampliación de la historia del concepto "experiencia", véase la Tesis Doctoral de Moreno (2012). 
abducción; el desarrollo de las consecuencias posibles de la hipótesis inicial, donde estaría la deducción; y la comprobación en la práctica de esas consecuencias, confirmando o desechando la hipótesis, esto es, la inducción. Por tanto, podríamos decir que la hipótesis es un proceso metacognitivo en el que se movilizan diferentes habilidades del pensamiento, como la contrastación, la analogía y la clasificación, entre otros. De esta manera, la lectura, la escritura, la búsqueda de información y el diálogo de saberes, contribuyen a los ajustes de la hipótesis formulada por los estudiantes y su profesor?.

\section{F. Aprendizajes socio-cognitivos y culturales}

De acuerdo con Zabala (2008, p. 55), "el modelo de investigación del medio", o lo que es lo mismo, la investigación en el aula, comprende las siguientes fases: motivación (creación o presentación de un problema [o hecho sorprendente]); preguntas (relacionadas con el problema) [o hipótesis]; selección de las fuentes de información y planificación de la investigación [o deducción]; generalización de las conclusiones [inducción]; y expresión y comunicación [o difusión de los hallazgos o investigación dialógica]8.

Sus planteamientos, se pueden recontextualizar en la construcción de una secuencia didáctica abductiva, la cual debe incluir una serie de unidades didácticas de investigación (UDI) que, al estar basadas en los tres tipos de razonamientos (abducción, deducción e inducción), le permiten al docente y a sus estudiantes responder al qué, al cómo y al para qué de la enseñanza y el aprendizaje9 9

\section{G. Diálogo interdisciplinar}

Para Tardif (2004), la lógica monodisciplinar con la que se forman los futuros docentes en las facultades

7 Para una mayor ampliación de las experiencias de investigación en el aula donde se formulan y desarrollan hipótesis, se recomienda ver el audiovisual "El taller de literatura investigativa como estrategia didáctica" (2008). También, un artículo de Moreno, Carvajal y Zapata (2009) y otro de Moreno, Carvajal y Arango (2012).

8 Las fases de motivación, preguntas, selección de fuentes y planeación, conclusiones y comunicación de los hallazgos, resumen el proceso para llegar a la formulación de una hipótesis o abducción creativa, como proceso de investigación en el aula.

9 Para una mayor ampliación de la manera como el docente puede potenciar las preguntas del qué, cómo y el para qué de los procesos de enseñanza y aprendizaje, se recomienda el artículo de Wojcikiewicz (2010), quien estudia las relaciones entre las categorías universales de experiencia de Peirce, como son: primeridad, segundidad y terceridad, y el concepto experiencias de aprendizaje de Dewey. de educación, presenta dos limitaciones: su énfasis en una disciplina convierte la ciencia en un saber fragmentado y especializado; mientras que su lógica privilegia la adquisición de conocimientos, poniendo en un segundo lugar sus cuestiones prácticas. Esto se evidencia en la falta de diálogo entre las disciplinas y en el diseño de las asignaturas. Estas últimas por lo general impulsan un conocimiento declarativo que no tiene ninguna relación con el futuro campo laboral de los estudiantes.

Por su parte, Barragán (2010), cuestiona el desinterés de algunos profesores por la docencia universitaria, cuando la consideran de un nivel inferior al de la investigación. Este divorcio entre la docencia y la investigación reproduce una visión unívoca y monodisciplinar de la ciencia, la cultura y la sociedad. Superar esta dificultad significa que debemos estar dispuestos a pensar un problema de nuestro campo de estudios desde la óptica de otra disciplina.

\section{H. Escritura}

En palabras de Gudmundsdottir (2005): "si sostenemos que el saber sobre los contenidos posee cualidades narrativas y pedagógicas, podemos comprender mejor su potencia heurística" (p. 59). La heurística, o arte de la creación de nuevas ideas, es precisamente lo que Peirce (2007) pretende proponer con su teoría de la abducción. Al respecto, señala que: "el desarrollo de la abducción es principalmente una cuestión de heurética" (p. 125). Por esta razón, consideramos que nuestra idea de encontrar en el método abductivo un proceso sociocognitivo y cultural para la investigación en el aula, encuentra en el saber narrativo del docente y los aprendizajes de investigación de los estudiantes, un enfoque adecuado para la reflexión escrita de sus hallazgos investigativos.

\section{Evaluación dialógica}

Para Blázquez (2002, pp. 341-368), el diálogo debe superar la visión instrumental con la que es mirado en la educación. Esto significa considerarlo como una "experiencia de liberación humana", un proceso a partir del cual sus interlocutores reconocen la presencia de diversos puntos de vista y guardan la intención de llegar a un acuerdo. Esta visión supone una revisión de las concepciones y las creencias del profesorado, debido al predominio que tiene la evaluación basada 
en el rendimiento académico. Esta "cultura dialogante" le exige a la institución educativa adelantar un cambio, expresado en una nueva concepción del ser humano y el quehacer educativo.

\section{J. Socialización}

El primer espacio de socialización de la experiencia de investigación es el salón de clases; allí puede surgir la idea de organizar un evento académico al interior de la institución educativa (escuela o universidad). Si estas instituciones están adscritas a las redes académicas o de conocimiento, la difusión de la experiencia puede ser más significativa debido a su impacto y su radio de acción, tanto local como global. La inclusión de los estudiantes y del profesorado en estas redes es un proceso a largo plazo.

Al respecto, Jurado et al. (2008), consideran que las redes académicas les brindan a sus integrantes la posibilidad de autoformarse y participar en la producción de conocimiento. Su interlocución es horizontal, al estar apoyadas en diversos medios electrónicos (blogs, portales, chats, teleconferencias, páginas Web, etc.) y en textos. Dichos textos "buscan esencialmente promover las interacciones a la manera de conversaciones textuales" (p. 53). Estas conversaciones textuales son una muestra de que el conocimiento y la información que circula en la escuela y en la universidad no son suficientes, pues el sujeto debe acudir a estos medios con la idea de buscar soluciones creativas a problemas comunes.

\section{K. Investigación dialógica}

Se entiende por investigación dialógica al proceso socio-cognitivo y cultural, a partir del cual el docente genera una serie de estrategias didácticas de investigación que hacen parte de su opción política y epistemológica. Dichas estrategias, promueven el diálogo de saberes entre los conocimientos inter y transdisciplinares del docente y las teorías de la pedagogía y la didáctica. Estos saberes también pueden ser experienciales, cuando el docente reflexiona su práctica pedagógica y la pone en discusión con sus pares académicos y los estudiantes. De esta manera, la investigación dialógica es un proceso para la reconstrucción del sentido, entre lo que se dice, se piensa, se sabe y se desea saber.
En este contexto, uno de los propósitos de la investigación dialógica se relaciona con la inclusión del profesorado, y sus estudiantes, en la cultura académica de la universidad. Al respecto, García (2008) señala que "no hay escritura sin lectura, y no hay investigación sin lectura y escritura". La interrelación de estos tres procesos debe hacer parte de los planes de formación de los futuros docentes, ya que en la escuela y en la universidad circulan textos académicos y discursos orales que responden a códigos y exigencias diferentes. Una de estas exigencias consiste en que el futuro maestro se haga consciente de que su ejercicio como lector y productor de textos, lo ubica en una posición pública y privada.

\section{Conclusiones}

Inicialmente, mostramos los problemas teóricoprácticos a los que se enfrenta la investigación en el aula, bien sea en la educación básica y media, como en la universidad. Señalamos que los aprendizajes de investigación, cuando parten del Deseo de saber de los estudiantes, y el docente los considera como elementos clave de su propuesta didáctica, se convierten en iniciativas de largo aliento en la vida de los estudiantes y en los procesos socio-cognitivos y culturales del aula.

Así mismo, expresamos que el enfoque constructivista y la red conceptual de la disciplina enseñada por el docente, favorecen los aprendizajes de investigación de los estudiantes de la educación básica y media, debido a su participación en la formulación de problemas, preguntas y proyectos que nacen de sus intereses. Por su parte, en la universidad, los conocimientos del profesor deben ser recontextualizados en su metódica para, así, potenciar en el aula las competencias científicas que la sociedad del conocimiento demanda de los futuros profesionales.

El esbozo de estos problemas y propuestas nos permitió sustentar que es posible pensar en una estrategia didáctica, representada en una Espiral Comprensiva de la Investigación en el Aula - ECEDIA-, como un proceso de investigación sustentado en tres principios didácticos: la experiencia dialógica, la conversación hermenéutica y el diálogo de saberes. Estos principios se sustentan en un marco referencial y un diseño metodológico.

En el primer caso, como veremos más adelante, la ECEDIA que fue representada por una serie de com- 
ponentes nodales, expone los enfoques que le bridan su consistencia teórica. En el segundo caso, el diseño metodológico muestra que la investigación cualitativa con enfoque hermenéutico, contribuye a establecer la relación entre el docente con sus estudiantes, y la de éstos con la ciencia, la cultura y la sociedad. Esto se expresa en los procesos de interrelación entre lo subjetivo y lo objetivo, el todo y las partes, el horizonte propio y el ajeno, la formulación de una hipótesis abductiva, entre otras. Esta concepción metodológica se proyecta en la investigación cuando se muestran los casos de aula donde se adelantó la construcción de la ECEDIA.

Uno de los casos, lo integra un grupo de maestros en formación de la Licenciatura en Humanidades, Lengua Castellana, de la Facultad de Educación de la Universidad de Antioquia. Su interacción con la ECEDIA le permitió formarse en algunas habilidades de la investigación y vivenciar la puesta en escena de su hipótesis abductiva, debido al diseño y desarrollo de sus proyectos de aula en instituciones de educación del nivel básico y medio.

El otro caso, está representado por dos grupos de profesores universitarios. El primero, de una universidad privada, y el segundo de una institución pública. El desarrollo de la primera experiencia comienza a mostrar que el énfasis de la estrategia didáctica en el texto literario, debía ser replanteado. Esto quiere decir, que una estrategia didáctica pensada para la educación superior, debía tener la capacidad de ponerse en escena en cualquier área de conocimiento. Máxime, cuando empezamos a observar que la hermenéutica, la abducción y el diálogo de saberes, o bien eran retomados de manera separada en diferentes disciplinas y ciencias, como por ejemplo, la hermenéutica en la investigación socioeducativa y la abducción en los estudios literarios; o se dejaba de lado la relación de estas teorías con el diálogo de saberes, no sólo en la Didáctica de la literatura, sino también en la Didáctica universitaria.

Esta desarticulación de saberes, empieza a sustentar la necesidad de crear la ECEDIA como una estrategia didáctica para la investigación en el aula. De esta manera, se muestra la representación mental de la ECEDIA, con la idea de que se observe el punto de partida (el diálogo de saberes) y los diez componentes nodales, que ponen en movimiento los procesos sociocognitivos y culturales de los estudiantes y del docente, cuando adelantan un proceso de indagación.
También mostramos que el rasgo distintivito de cada componente nodal es el siguiente: el diálogo de saberes, que le otorga al docente el papel de un traductor de los conocimientos que provienen de la ciencia y la cultura; la conversación hermenéutica, es el eje transversal de la ECEDIA, al posibilitar la creación de un ambiente de respeto y confianza entre el docente y sus estudiantes; y la historia de un concepto, que permite al docente actualizar el pasado de su saber y, de paso, promover el desarrollo de una conciencia histórica en sus estudiantes .

El hecho sorprendente, intenta rescatar la capacidad de asombro de los estudiantes y del docente como un estímulo socio-cognitivo y creativo, propio de nuestra condición como seres en evolución; la hipótesis abductiva, se convierte en un reto metacognitivo de los estudiantes y el docente, al exigirles la búsqueda de información para sustentar la posibilidad de su hallazgo.

Los aprendizajes socio-cognitivos y culturales de los estudiantes, al estar motivados por una hipótesis abductiva, producto de su Deseo de saber, deben organizarse por medio de una secuencia didáctica capaz de mostrar al docente y a sus estudiantes, los caminos que deben abrirse y reconfigurarse con base en sus horizontes de expectativas; el diálogo interdisciplinar, es un Ilamado a comprender que el desarrollo, los obstáculos de una ciencia y una disciplina, pueden ser analizados con el apoyo de otros conocimientos y saberes; la escritura, es una impronta del quehacer investigativo del docente y sus estudiantes, posibilitadora del desencuentro con el otro y lo otro, en busca de un acuerdo.

La evaluación dialógica, es una aspiración de la sociedad educadora, por tanto, avanzar en su concreción en el aula se convierte en un aporte al desarrollo de la humanidad; la socialización de la investigación, convierte al docente y a sus estudiantes en sujetos públicos dispuestos al diálogo razonado y a la construcción de acuerdos. La investigación dialógica, es la síntesis de la ECEDIA, pues allí confluyen dos propósitos: lograr que la investigación en la educación básica y media fortalezca la cultura escolar, en sus diversas dimensiones y ámbitos (socioculturales, estéticos, políticos, entre otros), y acercar a los estudiantes universitarios y a los profesores, a la comprensión de una cultura académica donde los significados de un texto tengan la posibilidad de convertirse en experiencias vitales y aprendizajes de investigación. 
Balbi Ochoa, A. (2008). "La investigación como estrategia didáctica en la acción docente". Kaleidoscopio, 5 (9). Venezuela: Universidad Nacional Experimental de Guayana, 21-31. Obtenida el 5 de Diciembre de 2010, desde, http://kaleidoscopio.uneg.edu. ve/numeros/k09/k09_art02.pdf

Blázquez Entonado, F. (2002). "La evaluación como proceso de diálogo". En Rodríguez, M. Rojo. (Coord.). Didáctica general. Qué y cómo enseñar en la sociedad de la información. Madrid: Biblioteca Nueva, 341-368.

Barragán Castrillón, B. (2010). "La enseñanza en la universidad: interdisciplinariedad, cultura académica y experiencia". Unipluridiversidad 10 (2). Medellín: Universidad de Antioquia, Facultad de Educación, 47-53.

García, J. E., y García, F. F. (1993). Aprender investigando: una propuesta metodológica basada en la investigación. Sevilla: Díada.

García, N. (2008, Julio-Diciembre). "Lectura, escritura, cultura académica y formación de docentes". Nodos y Nudos, No 25. Bogotá: Universidad Pedagógica Nacional, 81-91.

González A., Elvia María. (2005). "El desarrollo de competencias científicas en la educación superior". Lectiva, No. 10. Medellín: Producciones Colombianas.

González A., Elvia María. (2010). Sobre la experiencia hermenéutica o acerca de otra posibilidad para la construcción de conocimiento". Medellín: Universidad de Antioquia, Facultad de Educación, Doctorado en Educación. Manuscrito no publicado.

Gudmundsdottir, S. (2005). "La naturaleza narrativa del saber pedagógico sobre los contenidos". En: McEwan, H., y Egan. K. (Comps.). La narrativa en la enseñanza y el aprendizaje y la investigación. Buenos Aires: Amorrortu, 52-71.

Gurdián Fernández, Alicia. (2007). El paradigma cualitativo en la investigación socio-educativa. San José de Costa Rica: Colección IDER. Coordinación Educativa y Cultural Centroamericana (CECC), Agencia Española de Cooperación Internacional (AECI).

Gadamer, Hans Georg. (2004). Verdad y método II. Traducción M. Olasagastitrad. Salamanca: Sígueme.

Gadamer, Hans Georg. (2005). Verdad y método I. Trad. A. Agud Aparicio y R. de Agapito. Salamanca: Sígueme.

Jurado, F., González, S., y Rodríguez. E. (2008). "La evaluación educativa y la pertinencia de las redes". En: Cardona, M., y García, H. (Comps.). Educación superior hoy. Algunas reflexiones y retos. Bogotá: ICFES, Grupo de Investigación en Evaluación y Calidad de la Educación (GIECE), Semillero de Investigación en Evaluación y Calidad de la Educación (SIECE), Universidad San Buenaventura, 52-59.
Maturana Romesín, Humberto. (1997). La realidad: ¿objetiva o construida? México: Anthropos. Primera parte, pp. 3-102. Segunda parte, pp.105-159.

Moreno Torres, M. (2008). "El taller de literatura investigativa como estrategia didáctica". Audiovisual editado por la Facultad de Educación de la Universidad de Antioquia, Línea Doctoral Didáctica Universitaria. Duración 20 minutos.

Moreno Torres, M., Carvajal C. E., y Zapata, C. (2009). "Diseño de un proyecto didáctico de literatura como abducción creativa y visión horizóntica". Nodos y Nudos, 3 (27), 43-55.

Moreno T., Carvajal, M., y Arango, Y. (2012). "La hipótesis abductiva como estrategia didáctica de investigación en el aula". Manuscrito para publicación, Revista Ikala, Medellín. Obtenido desde http://aprendeenlinea.udea.edu.co/revistas/index.php/ikala

Moreno T., Carvajal, M., y Arango, Y. (2012). "Fundamentación de una estrategia didáctica basada en la teoría de la abducción, la hermenéutica y el diálogo de saberes para la formación de profesores investigadores en la educación básica, media y superior". Tesis para optar al título de Doctora en Educación. Medellín: Universidad de Antioquia, Facultad de Educación, Centro de Documentación.

Nubiola, J. (2001, Febrero-Abril). "La abducción o lógica de la sorpresa". Razón y Palabra N. ${ }^{\circ} 21$. Obtenido el 29 de Junio de 2011, desde http://www.razonypalabra.org.mx/anteriores/ n21/21_jnubiola.html

Peirce, C. S. (1901). "El tratamiento apropiado de las hipótesis". Traducción R. de Narváez (2009). Obtenido el 30 de Marzo de 2010, desde http://www.unav.es/gep

Ruiz Bolívar, C., y Torres Pacheco, V. (2005). "La enseñanza de la investigación en la universidad: el caso de una universidad pública venezolana". Investigación y Postgrado, Vol. 20 N. ${ }^{\circ}$, 13-34. Obtenido el 31 de Agosto de 2008, desde http://www. scielo.org.ve/scielo.php?script=sci_arttex\&pid=S1316

Tardif, M. (2004). Los saberes del docente y su desarrollo profesional. Madrid: Narcea.

Wojcikiewicz, S. K. (2010). “Dewey, Peirce y las categorías de aprendizaje". Traducción H. Schmidt. Education and Culture, Vol. 26, No 2. Purdue University Press, 65-82.

Zabala Vidella, A. (2008). La práctica educativa. Cómo enseñar. Barcelona: Graó, 53-90. 


\section{Diálogo del conocimiento}

Este artículo aporta en la comprensión, reflexión y búsqueda de estrategias didácticas para pensar la investigación del maestro y la investigación del estudiante en los diferentes niveles de la escolaridad, en particular en la educación superior, lo cual se constituye en un reto y en un desafío para los maestros que están buscando permanentemente opciones y alternativas para contribuir en la formación de competencias investigativas de los estudiantes. Lo cual se evidencia en el presente artículo, en el que se significa y resignifica los procesos investigativos en el aula visibilizando la actividad de la maestra quien da cuenta de la desarticulación entre el saber disciplinar, el saber didáctico y los aprendizajes de investigación de los estudiantes. El origen y la elección del tema-problema objeto de investigación puede emerger de la maestra o del estudiante o de un interés conjunto planteado como una pregunta o un problema.

Un asunto relevante en la actividad de la maestra investigadora, es la distinción de la perspectiva y enfoque investigativo y de la elección de técnicas y herramientas para la recolección de la información que posibiliten dar cuenta del objeto de investigación, para el caso que se socializa "construir una estrategia didáctica interesada en promover algunas habilidades de investigación en los estudiantes", en el cual se opta por la investigación cualitativa con enfoque hermenéutico, siendo coherente con las consideraciones teórico-metodológicas de la investigación.

En su búsqueda la autora considera que es posible realizar procesos de investigación en el aula donde se articulen las preocupaciones teóricas del profesorado (saber disciplinar y saber didáctico) a los aprendizajes de investigación de sus estudiantes, lo cual se visibiliza en la apuesta de la estrategia didáctica la Espiral Comprensiva de la Experiencia de Investigación en el Aula -Ecedia- soportada en los principios de una experiencia dialógica, la interacción pedagógica dada por la conversación hermenéutica, y que el conocimiento de la ciencia y la cultura en están basados en el diálogo de saberes.

La Ecedia se constituye en la apuesta de la investigación por un lado, porque tiene sentido para los maestros en formación del área de lengua y literatura, y por otro, porque se constituye en un aporte a los procesos de investigación en la educación superior. Los componentes de la Ecedia, el diálogo de saberes, la conversación hermenéutica, la historia de conceptos, el hecho sorprendente, la hipótesis abductiva, los aprendizajes socio-cognitivos y culturales, el diálogo interdisciplinar, la escritura, la evaluación dialógica, la socialización y la investigación dialógica, dan cuenta de los referentes, miradas y sentidos al pensar en la búsqueda de estrategias didácticas de investigación en la educación superior, en particular basada en el diálogo de saberes.

La propuesta del trabajo en el aula y la realización de investigaciones por parte del docente basados en el diálogo de saberes permite reconocer las diferentes perspectivas e intereses de los estudiantes en los encuentros en la clase, distinguir opciones para generar los procesos investigativos en el aula y enriquecer el bagaje conceptual del docente investigador. Actividad de gran importancia para la comunidad académica dado que permite la distinción, reconocimiento y diferenciación de perspectivas, tendencias o énfasis para pensar la formulación de investigaciones y estrategias para formar estudiantes investigadores, en particular en la educación superior.

Rosa Inés Pedreros Martínez 